Fukushima J. Med. Sci.,

Vol. 63, No. 3, 2017

[Original Article]

\title{
The incidence and severity of IgA vasculitis with nephritis over a 10-year period in our hospital
}

\author{
Keishi Yamane, Yukihiko Kawasaki, Ryo Maeda, Shinichiro Ohara, \\ Kazuhide Suyama and Mitsuaki Hosoya \\ Department of Pediatrics, Fukushima Medical University School of Medicine
}

(Received June 12, 2017, accepted August 17, 2017)

\begin{abstract}
Background : To evaluate the recent frequency of onset and severity of IgA vasculitis with nephritis (IgAVN) in Fukushima Prefecture, we examined the epidemiology and clinico-pathological manifestations of IgAVN in our hospital over a 10-year period.

Methods : We enrolled 18 patients with IgAVN treated between 2004 and 2013 in the Department of Pediatrics, Fukushima Medical University School of Medicine. These patients were divided into two groups ; Group 1 consisted of 12 patients with IgAVN hospitalized between 2004 and 2008 and Group 2 consisted of 6 patients with IgAVN hospitalized between 2009 and 2013. The epidemiology, clinical features, laboratory data, pathological findings, and outcome were retrospectively compared between the two groups.

Results : The numbers of patients with IgAVN per year in Group 2 were lower than that in Group 1. The frequency of patients with higher than grade IIIb disease in Group 2 (50\%) was lower than that in Group $1(94 \%)$; furthermore, the frequency of patients with higher than grade IV disease in Group 2 (0\%) was lower than that in Group $1(50 \%)$.

Conclusions : Our findings suggest that the incidence of onset and severity of IgAVN in patients diagnosed between 2009 and 2013 were lower than those in patients diagnosed between 2004 and 2008.
\end{abstract}

Key words : IgA vasculitis with nephritis, epidemiology, steroid, bredinine, children

\section{Introduction}

IgA vasculitis (IgAV) is an immunoglobulin (Ig) A-mediated immune-complex vasculitis affecting predominantly the skin, joints, gastrointestinal tract, and kidneys. Previously known as Henoch-Schönlein purpura, it is a form of systemic small vessel vasculitis that occurs most frequently in childhood, with the prognosis for IgAV patients largely predicted by the severity of renal involvement ${ }^{1,2)}$.

The epidemiology of IgAV is obscure and the proportion of patients reported to have renal involvement varies between $30 \%$ and $60 \%{ }^{1-5)}$. Recently, Trapani et al. reported that 77 (54\%) of 150 patients with IgAV had renal involvement, 11 (7\%) had severe nephritis, and $3(2 \%)$ had acute renal insufficiency ${ }^{3)}$. The majority of children with IgA vasculitis with nephritis (IgAVN) presenting with only hematuria and/or low-grade proteinuria have a good chance of recovery. However, patients with massive proteinuria at onset frequently show a progressive course $\mathrm{c}^{5-9)}$.

As to the changes in the onset rate of IgAVN in Japan, Moriya et al. ${ }^{10)}$ investigated 30 patients diagnosed with IgAVN between 1971 and 1982 and 11 patients diagnosed with IgAVN between 1985 and 1996, and found that the severity of the renal histopathological findings was lower in the 11 patients with IgAVN diagnosed between 1985 and 1996. Further, we investigated the clinic-pathological find-

Corresponding author : Yukihiko Kawasaki E-mai : kyuki@fmu.ac.jp

https://www.jstage.jst.go.jp/browse/fms http://www.fmu.ac.jp/home/lib/F-igaku/ 
ings in $120 \mathrm{HSPN}$ patients and found that the incidence and severity of IgAVN in patients hospitalized between 1987 and 1997 were similar to those in patients hospitalized between 1998 and 2008 and that the proportion of patients with severe IgAVN had not decreased. However, apart from the above report, there have been no reports on the changes in the onset rate of IgAVN during the 2000s and few recent reports on the decrease in hospitalization rates among IgAV patients ${ }^{11}$. Thus, we investigated the epidemiology and clinical manifestations of IgAVN over a 10 -year period in our hospital to clarify the recent changes in the incidence ; and severity of IgAVN in patients.

\section{Patients and Methods}

The study was carried out under the auspices of the Committee for Human Experiments at the Fukushima Medical University School (Institutional Review Board approval number 2253). Informed consent was obtained from all patients or their parents.

\section{Patients}

We enrolled 18 patients diagnosed with IgAVN between 2004 and 2013 in the Department of Pediatrics, Fukushima Medical University School of Medicine. These patients were divided into two groups. Group 1 consisted of 12 patients with IgAVN hospitalized between 2004 and 2008 and Group 2 consisted of 6 patients with IgAVN hospitalized between 2009 and 2013. The epidemiology, clinical features, laboratory data, pathological findings, treatment, and outcome were retrospectively compared between Group 1 and Group 2.

\section{Definitions}

Hematuria was regarded as positive if microscopic examination showed five or more red blood cells per high-power field, and macrohematuria was diagnosed if the same number of red blood cells were visible with the naked eye ${ }^{11}$. Proteinuria was evaluated by 24 -hour quantitative measurement. Nephrotic syndrome was defined as the presence of proteinuria ( $\geqq 40 \mathrm{mg} / \mathrm{m}^{2} / \mathrm{h}$ ) and a serum albumin level of less than $2.5 \mathrm{~g} / \mathrm{dl}$, with or without ede$\mathrm{ma}^{11,12)}$. A diagnosis of IgAV was made if the major manifestations of the illness consisted of a purpuric rash and abdominal pain without thrombocytopenia. Additional features including arthritis and nephritis were accepted as being consistent with the diagnosis.
The clinical status of each patient at the latest observation was classified as follows.

Stage 1) normal : the patient was normal on physical examination, with normal urine and renal function.

Stage 2) minor urinary abnormalities : the patient was normal on physical examination, with microscopic hematuria or proteinuria of less than 20 $\mathrm{mg} / \mathrm{m}^{2} / \mathrm{h}$.

Stage 3) persistent nephropathy : the patient had proteinuria of $20 \mathrm{mg} / \mathrm{m}^{2} / \mathrm{h}$ or greater or hypertension, and twenty-four hour creatinine clearance (24hCcr) of $60 \mathrm{ml} / \mathrm{min} / 1.73 \mathrm{~m}^{2}$ or greater.

Stage 4) renal insufficiency : the patient had $24 \mathrm{hCcr}$ less than $60 \mathrm{ml} / \mathrm{min} / 1.73 \mathrm{~m}^{2}$ (including dialysis/transplant or death).

\section{Pathology}

First renal biopsies were performed for all patients. The specimens were assessed by light microscopy (LM) and immunofluorescence (IF). Material for histological study was fixed in $12 \%$ neutral formalin, and embedded in paraffin, sectioned at a thickness of 2-3 $\mu \mathrm{m}$, and stained with hematoxylin and eosin or Periodic acid-Schiff reagent.

The glomerular changes were graded according to the classification devised by the pathologists of the International Society of Kidney Disease in Children $^{13)}$, as follows : I. minor glomerular abnormalities ; II. pure mesangial proliferation [(a) focal, (b) diffuse] ; III minor glomerular abnormalities or mesangial proliferation, with crescents in $<50 \%$ of glomeruli [(a) focal, (b) diffuse mesangial proliferation] ; IV, same as III but with crescents in 50\%$75 \%$ of glomeruli [(a) focal, (b) diffuse mesangial proliferation] ; V same as III but with crescents in $>75 \%$ glomeruli [(a) focal, (b) diffuse mesangial proliferation] ; VI, membrano-proliferative-like lesions.

\section{Treatment}

Following diagnostic renal biopsy, the patients with grade I or II findings were treated with antiplatelet agents(dipyridamole $5 \mathrm{mg} / \mathrm{kg} /$ day), while patients with grade IIIa findings were treated with daily oral prednisolone $(1 \mathrm{mg} / \mathrm{kg} /$ day $)$ for a period of more than 6 months, along with anti-platelet agents and warfarin. Warfarin was given orally in a single morning dose of $1 \mathrm{mg}$ (for patients aged less the 7 years) or $2 \mathrm{mg}$ (for patients aged 7 years or over) to maintain the thrombotest level at 30 to $50 \%$. Patients with grade IIIb findings were treated with MUP therapy. MUP therapy consisted of a combi- 
nation of pulse methylprednisolone at $30 \mathrm{mg} / \mathrm{kg} /$ day i.v. bolus (maximum $1 \mathrm{~g}$ ) for three consecutive days, and pulse urokinase at $5,000 \mathrm{u} / \mathrm{kg}$ /day i.v. bolus (maximum 180,000 u) for seven consecutive days, followed by daily oral prednisolone $(1 \mathrm{mg} / \mathrm{kg} /$ day $)$ for a period of more than 6 months, along with antiplatelet agents and anti-coagulant ${ }^{15)}$. Patients with grade VI or higher findings were treated with MUP therapy plus cyclophosphamide. Cyclophosphamide was given orally at $2.5 \mathrm{mg} / \mathrm{kg} /$ day for 12 weeks ${ }^{16)}$. Patients graded higher than ISKDC grade $\mathrm{V}$ were treated with plasmapheresis $(\mathrm{PP})^{17)}$.

\section{Statistics}

Data are expressed as mean values \pm SEM. Statistical analysis was performed on a Macintosh computer using a Stat View software package (Abacus Concepts, Berkeley, Calif., USA). Several variables, which were clearly not in a normal distribution, were compared using non-parametric statistics such as Mann-Whitney' test or Wilcoxon test. A p value $<0.05$ was considered significant.

\section{Results}

1) Comparison of patient's characteristics at the onset of symptoms between groups (Table 1, 2, Figure 1)

Age at onset (years) and male-to-female ratio were $9.0 \pm 3.5$ and $5 / 7$, respectively, in Group 1 , and $7.8 \pm 1.5$ and $3 / 3$ in Group 2. The numbers of patients diagnosed with IgAVN per year was $2.4 \pm 0.8$ in Group 1 and $1.2 \pm 0.8$ in Group 2, and the numbers of patients diagnosed with IgAVN per year in Group 2 was lower than that in Group 1 [Figure 1]. The duration from onset to biopsy (months) was $1.9 \pm$ 1.6 in Group 1 and $3.1 \pm 4.4$ in Group 2, with no significant difference observed between groups.

Among the clinical manifestations at onset in Group 1 and Group 2, the most common clinical symptoms were purpura (in $100 \%$ of patients), abdominal pain (83\%), arthralgia (56\%), nephrotic syndrome $(44 \%)$, and again there were no significant differences in the clinical manifestations at onset between the two groups (Table 1). On the basis of laboratory data, no differences were observed in urinary protein excretion, the frequency of hematuria, serum albumin levels, serum creatinine levels or $\mathrm{e}^{-}$ GFR between the two groups (Table 2).

2) Comparison of pathological findings between groups at the time of the first renal biopsy (Table 3)

LM findings at first renal biopsy revealed that the most common ISKDC classification was grade III b (in $44 \%$ of patients), grade III a $(22 \%)$, grade IV $(6 \%)$, grade V $(11 \%)$, grade VI $(17 \%)$ and the number of patients with grade III a disease in Group 2 was higher than that in Group $1(\mathrm{p}<0.05)$. The frequency of patients with higher than grade III $\mathrm{b}$ disease in Group $2(50 \%)$ was lower than that in Group $1(94 \%)(\mathrm{p}<0.05)$; furthermore, the frequency of patients with higher than grade IV disease in Group $2(0 \%)$ was lower than that in Group $1(50 \%)$ $(\mathrm{p}<0.05)$.

Table 1. Comparison of clinical manifestations at onset between Group 1 and Group 2

\begin{tabular}{l|c|c|c}
\hline & $\begin{array}{c}\text { Total } \\
(\mathrm{n}=18)\end{array}$ & $\begin{array}{c}\text { Group 1 } \\
(\mathrm{n}=12)\end{array}$ & $\begin{array}{c}\text { Group 2 } \\
(\mathrm{n}=6)\end{array}$ \\
\hline Purpura & $18(100 \%)$ & $12(100 \%)$ & $6(100 \%)$ \\
Abdominal pain & $15(83 \%)$ & $11(92 \%)$ & $4(67 \%)$ \\
Arthralgia & $10(56 \%)$ & $6(50 \%)$ & $4(67 \%)$ \\
Trias & $8(44 \%)$ & $6(50 \%)$ & $2(33 \%)$ \\
Purpura only & $2(11 \%)$ & $1(8 \%)$ & $1(17 \%)$ \\
Nephrotic syndrome & $8(44 \%)$ & $6(50 \%)$ & $2(33 \%)$ \\
\hline
\end{tabular}

Table 2. Comparison of laboratory data at the time of the first biopsy between Group 1 and Group 2

\begin{tabular}{l|c|c|c}
\hline & $\begin{array}{c}\text { Total } \\
(\mathrm{n}=18)\end{array}$ & $\begin{array}{c}\text { Group 1 } \\
(\mathrm{n}=12)\end{array}$ & $\begin{array}{c}\text { Group 2 } \\
(\mathrm{n}=6)\end{array}$ \\
\hline Urinary protein excretion (g/day) & $2.7 \pm 2.5$ & $3.4 \pm 2.8$ & $1.3 \pm 0.9$ \\
Hematuria case $(\%)$ & $18(100)$ & $12(100)$ & $6(100)$ \\
Serum albumin $(\mathrm{mg} / \mathrm{dl})$ & $3.3 \pm 0.7$ & $3.3 \pm 0.7$ & $3.3 \pm 0.7$ \\
Serum creatinine $(\mathrm{mg} / \mathrm{ml})$ & $0.38 \pm 0.11$ & $0.39 \pm 0.13$ & $0.34 \pm 0.04$ \\
eGFR $\left(\mathrm{ml} / \mathrm{min} / 1.73 \mathrm{~m}^{2}\right)$ & $126 \pm 27$ & $125 \pm 32$ & $128 \pm 14$ \\
\hline
\end{tabular}




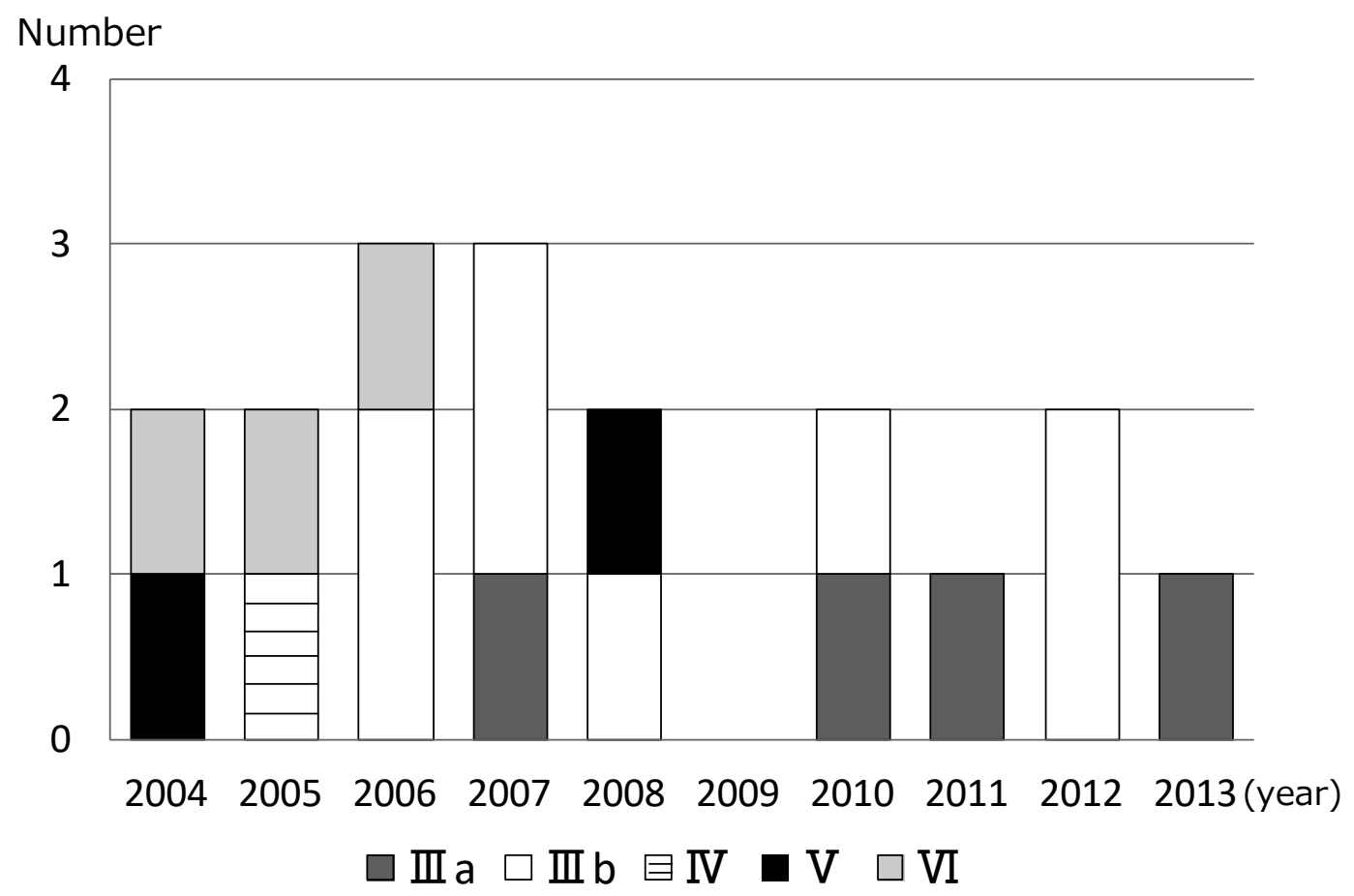

Fig. 1. Comparison of the numbers of patients diagnosed with IgA vasculitis with nephritis each year in the two groups.

Table 3. Comparison of ISKDC classification between Group 1 and Group 2

\begin{tabular}{c|c|c|c|c}
\hline & $\begin{array}{c}\text { Total } \\
(\mathrm{n}=18)\end{array}$ & $\begin{array}{c}\text { Group 1 } \\
(\mathrm{n}=12)\end{array}$ & $\begin{array}{c}\text { Group 2 } \\
(\mathrm{n}=6)\end{array}$ & \\
\hline II & $0(0 \%)$ & $0(0 \%)$ & $0(0 \%)$ & N.S. \\
IIIa & $4(22 \%)$ & $1(8 \%)$ & $3(50 \%)$ & $\mathrm{p}<0.05$ \\
IIIb & $8(44 \%)$ & $5(42 \%)$ & $3(50 \%)$ & N.S. \\
IV & $1(6 \%)$ & $1(8 \%)$ & $0(0 \%)$ & N.S. \\
V & $2(11 \%)$ & $2(17 \%)$ & $0(0 \%)$ & N.S. \\
VI & $3(17 \%)$ & $3(25 \%)$ & $0(0 \%)$ & N.S. \\
\hline
\end{tabular}

N.S., Not significant
3) Comparison of treatment and clinical stage at 2 years after treatment between groups. (Table 4)

All patients in Group 1 and Group 2 were treated with prednisolone and anti-platelet agents, 10 patients (83\%) in Group 1 and 5 (83\%) in Group 2 were treated with MUT therapy, 4 patients (33\%) in Group 1 and none in Group 2 were treated with cyclophosphamide, and 4 patients (5\%) in Group 1 and none in Group 2 were treated with plasmapheresis. In addition, 4 patients (33\%) in Group 1 and 4 patients $(67 \%)$ in Group 2 were treated with mizoribine and one patient in Group 1 was treated with cyclosporine.

Table 4. Comparison of the treatments and the clinical stage at 2 years after treatment between Group 1 and Group 2

\begin{tabular}{ll|c|c|c|c}
\hline & \multicolumn{1}{|c|}{$\begin{array}{c}\text { Total } \\
(\mathrm{n}=18)\end{array}$} & $\begin{array}{c}\text { Group } 1 \\
(\mathrm{n}=12)\end{array}$ & $\begin{array}{c}\text { Group } 2 \\
(\mathrm{n}=6)\end{array}$ & \\
\hline Treatments & Prednisolone & $18(100 \%)$ & $12(100 \%)$ & $6(100 \%)$ & N.S \\
& Anti-platelet agents & $18(100 \%)$ & $12(100 \%)$ & $6(100 \%)$ & N.S \\
& MUT therapy & $15(83 \%)$ & $10(83 \%)$ & $5(83 \%)$ & N.S \\
& Cyclophosphamide & $4(22 \%)$ & $4(33 \%)$ & $0(0 \%)$ & N.S \\
& Mizoribine & $8(44 \%)$ & $4(33 \%)$ & $4(67 \%)$ & N.S \\
& Cyclosporine & $1(6 \%)$ & $1(8 \%)$ & $0(0 \%)$ & N.S \\
\hline \multirow{2}{*}{ Clinical stage } & Stage 1 & $15(83 \%)$ & $11(92 \%)$ & $4(67 \%)$ & N.S \\
& Stage 2 & $3(17 \%)$ & $1(8 \%)$ & $2(33 \%)$ & N.S \\
& Stage 3 & $0(0 \%)$ & $0(0 \%)$ & $0(0 \%)$ & N.S \\
& Stage 4 & $0(0 \%)$ & $0(0 \%)$ & $0(0 \%)$ & N.S \\
\hline
\end{tabular}

N.S., Not significant 
As to the clinical stage at 2 years after treatment, $11(92 \%)$ patients in Group 1 and $4(67 \%)$ patients in Group 2 had clinically recovered, $1(8 \%)$ in Group 1 and 2 (33\%) in Group 2 patients had minimal urinary abnormalities, an no patients in Group 1 or Group 2 had active renal disease or renal insufficiency.

\section{Discussion}

With regard to the incidence of IgAV, Piram prospectively examined IgAV patients in Val de Marne county, a suburb of Paris, with 263874 residents $<15$ years old from 2012 to 2014 and identified 147 incident cases, indicating an annual incidence of 18.6 per 100,000 children ${ }^{18)}$. Gardner-Medwin et al. ${ }^{19)}$ examined frequency of and ethnic variation in childhood vasculitis in the West Midlands region of the United Kingdom and reported that the annual incidence of IgAV was 22.1 per 100,000 , which was higher than the previous estimates of 13.5 to 18.0 per $100,000^{20)}$. Gardner-Medwin et al. postulated that a higher incidence of IgAV may lead to increases in the incidence of renal disease and need for renal medical treatment.

Regard with the incidence of IgAVN, it was reported that the mean number of IgAVN patients per 100,000 children per year was 4.9 in Asian children $^{19)}$. In Japan, it was reported that the number of HSPN patients or the number of severe HSPN were decreased from 1971 to $1996^{10)}$. Thus, we previously investigated the incidence and severity of IgAVN patients from 1987 to 2008 in Fukushima prefecture and found the number of HSPN patients or the number of severe HSPN were not decreased ${ }^{11)}$. However, there have been few reports on the decrease in hospitalization rates among IgAV patients $^{21}$. Okubo et al. reported that the total annual hospitalization rates for IgAV showed a significant decrease, ranging from 2.45 per 100,000 children in 2003 to 1.89 per 100,000 children in $2012(\mathrm{p}<0.001)^{20)}$. Our study, which was based on a follow-up of patients over a 10 -year periods, revealed that the number of IgAV patients hospitalized from 2009 to 2013 in Fukushima prefecture was less than that of IgAV patients hospitalized between 2004 and 2008. This finding suggested that the incidence of IgAVN in the second five-year period of our study had decreased. As the duration of our observation was rather short, it is necessary to observe the changes in the number of IgAV or IgAVN over a long-term follow up.

For classification of the degree of renal involve- ment and its correlation with clinical manifestations and prognostic indices, glomerular changes were graded according to the classification devised by the pathologists of the International Society of Kidney Disease (ISKDC) in children ${ }^{2}$. Patients with grade II and IIIa histological findings tend to have better outcomes, with either return of normal renal function or persistent microscopic hematuria and proteinuria, whereas patients with grade IIIb, IV, and V have persistent proteinuria and hematuria or progress to terminal renal failure3. A few patients develop rapidly progressive renal failure accompanied by exuberant crescent formation.

In our study, the frequency of patients with more than grade IIIb disease in Group $2(50 \%)$ was lower than that in Group 1 (94\%), with the frequency of patients with higher than grade IV disease in Group $2(0 \%)$ also lower than that in Group 1 (50\%). Thus, the frequency of severe HSPN patients hospitalized from 2009 to 2013 in Fukushima prefecture was lower than that of HSPN patients hospitalized between 2004 and 2008.

As to the clinical stage at 2 years after treatment, all patients in Group 1 and Group 2 had clinically recovered or minimal urinary abnormalities. The outcome in our hospital at 2 years after treatment was good in both groups.

In conclusion, our findings suggest that the incidence of onset and severity of IgAVN in patients hospitalized between 2004 and 2008 were lower those in patients hospitalized between 2009 and 2013. However, it is difficult at this time to emphasize that the number and severity of IgAVN patients has decreased from a small, retrospective study conducted at just one institution. It will, of course, be necessary to further evaluate the changes in the frequency of onset and severity of IgAVN through prospective studies at a larger number of institutions.

\section{Acknowledgments}

The authors thank the members of the Department of Pediatrics for their valuable advice and comments in relation to this study. None of the authors have financial relationships with any companies with an interest in the subject matter of this manuscript.

\section{References}

1. Jennette JC, Falk RJ, Bacon PA, et al. 2012 revised International Chapel Hill Consensus Conference Nomenclature of Vasculitides. Arthritis Rheum, 65(1) : 1-11, 2013. 
2. Habib R, Niaudet R, Levy M. Schönlein-Henoch purpura nephritis and IgA nephropathy. Renal pathology with clinical and functional correlations, $2^{\text {nd }}$ edn. Lippincott, Philadelphia, Tisher CC, Brenner BM ; 427-523 1994.

3. Trapani S, Micheli A, Grisolia F, et al. HenochSchönlein purpura in childhood : epidemiological and clinical analysis of 150 cases over a 5-year period and review of literature. Semin Arthritis Rheum, 35(3) : 143-153, 2005.

4. Fretzayas A, Sionti I, Moustaki M, et al. HenochSchönlein purpura : a long-term prospective study in Greek children. J Clin Rheumatol, 14(6) : 324-331, 2008.

5. Haycock GB. The nephritis of Henoch-Schönlein purpura. Oxford Textbook of Nephrology, $2^{\text {nd }}$ edn. Oxford University Press, Oxford, Cameron JS 585-612,1998.

6. Niaudet P, Habib R. Methylprednisolone pulse therapy in the treatment of severe forms of Schönlein-Henoch purpura nephritis. Pediatr Nephrol, 12(3) : 238-243,1998.

7. Watanabe T, Takahashi S, Nakajo S, et al. Pathological improvement of IgA nephropathy and Henoch-Schönlein purpura nephritis with urokinase therapy. Acta Paediatrica Japonica, 38(6) : 622628, 1996.

8. Coppo R, Mazzucco G, Cagnoli L, et al. HenochSchönlein purpura Long-term prognosis of Henoch-Schönlein nephritis in adults and children. Nephrol Dial Transplant, 12(11) : 2277-2283, 1997.

9. Counahan R, Winterborn MH, White RHR, et al. Prognosis of Henoch-Schönlein nephritis in children. Br Med J, 2(6078) : 11-14, 1977.

10. Moriya S, Iitaka K, Koshino H, et al. HenochSchönlein purpura nephritis (HSPN) in children : comparison of the incidence and severity between two 12-year groups. Nippon Jinzo Gakkai Shi, 42(1) : 36-40,2000.

11. Kawasaki Y, Suyama K, Yugeta E, et al. The incidence and severity of Henoch-Schoenlein purpura nephritis over a 22 -year period in Fukushima Prefecture, Japan. Int Urol Nephrol, 42(4), 10231029, 2010.

12. Southwest Pediatric Nephrology Study Group. A clinico-pathologic study of crescentic glomerulonephritis in 50 children : A report of the Southwest Pediatric Nephrology Study Group. Kidney Int, 27(2) : 450-458, 1985.

13. National Heart, Lung and Blood Institute, Bethes- da, Maryland. Report of the Second Task Force on Blood Pressure Control in children. Pediatrics, 79(1) : 1-25, 1987.

14. White RHR. Henoch-Schönlein nephritis. A disease with significant late sequelae. Nephron, 68(1) : 1-9, 1994.

15. Kawasaki Y, Suzuki J, Nozawa R, et al. Efficacy of methylprednisolone and urokinase pulse therapy for severe Henoch-Schönlein nephritis. Pediatrics, 111(4) : 785-789, 2003.

16. Kawasaki Y, Suzuki J, Suzuki H. Efficacy of methylprednisolone and urokinase pulse therapy combined with or without cyclophosphamide in severe Henoch-Schönlein : a clinical and histopathological study. Nephrol Dial Transplant, 19(4) : 858-864, 2004.

17. Hattori M, Ito K, Konomoto T, et al. Plasmapheresis as the Sole Therapy for Rapidly Progressive Henoch-Schönlein Purpura Nephritis in Children. Am J Kidney Dis, 33(3) : 427-433, 1999.

18. Piram M, Maldini C, Biscardi S, De Suremain N, Orzechowski C, Georget E, Regnard D, Koné-Paut I, Mahr A. 1. Incidence of IgA vasculitis in children estimated by four-source capture-recapture analysis : a population-based study. Rheumatology (Oxford). 2017 Apr 24. doi:10.1093/rheumatology/ kex158. [Epub ahead of print]

19. Gardner-Medwin JMM, Dolezalova P, Cummins C, et al. Incidence of Henoch-Schönlein purpura, Kawasaki disease, and rare vasculitides in children of different ethnic origins. The Lancet, 19 ; 360(9341) : 1197-1202,2002.

20. Nielsen HE. Epidemiology of Schönlein-Henoch Purpura. Acu Pathol Scand 77(1) : 125-131, 1988.

21. Okubo Y, Nochioka K, Sakakibara H, et al. Nationwide epidemiological survey of childhood IgA vasculitis associated hospitalization in the USA. Clin Rheumatol, 35(11) : 2749-2756, 2016.

22. Iijima K, Ito-Kariya S, Nakamura H, et al. Multiple combined therapy for severe Henoch-Schönlein nephritis in children. Pediatr Nephrol, 12(3) : 244-248, 1998.

23. Gianviti A, Trompeter RS, Barratt TM, et al. Retrospective study of plasma exchange in patients with idiopathic rapidly progressive glomerulonephritis and vasculitis. Arch Dis Child, 75(3) : 186-190, 1996.

24. Kawasaki Y. The pathogenesis and treatment of pediatric Henoch-Schönlein purpura nephritis, Clin Exp Nephrol, 15(5) : 648-657, 2011. 